\title{
Coupled Gravitational Fields A New Paradigm for Propulsion Science
}

\author{
Walter Dröscher ${ }^{1}$, Jochem Hauser ${ }^{2 *}$ \\ ${ }^{1}$ Institut für Grenzgebiete der Wissenschaft, 6010 Innsbruck, Austria \\ ${ }^{2}$ Faculty H, Ostfalia Univ. of Applied Sciences, Campus Suderburg, 29556, Germany
}

\begin{abstract}
Current space transportation systems are based on the principle of momentum conservation of classical physics. Therefore, all space vehicles need some kind of fuel for their operation. The basic physics underlying this propulsion principle severely limits the specific impulse and/or available thrust. Launch capabilities from the surface of the Earth require huge amounts of fuel. Hence, space flight, as envisaged by von Braun in the early 50 s of the last century, has not been possible due to this concept. Only with novel physical principles, providing the proper engineering principles for propellantless propulsion, can these limits be overcome. The concept of gravitational field propulsion represents such a novel principle, not being based on the movement of extremely large masses (e.g., planets or stars), but by the capability of building devices for the generation of gravity-like (i.e. acceleration) fields in a way similar to electromagnetism. In other words, gravity fields should be technically controllable. Since a propulsion system based on gravity-like fields has to function in empty space, it has to interact with the spacetime field itself. At present, physicists believe that there are four fundamental interactions: strong (nuclei, short range), weak (radioactive decay, short range), electromagnetic (long range), and gravitational (long range). As experience has shown over the last six decades, none of these physical interactions is suitable as a basis for novel space propulsion. Furthermore, none of the advanced physical theories, like string theory or quantum gravity, go beyond these four known interactions. On the contrary, recent results from causal dynamical triangulation simulations indicate that wormholes in spacetime do not seem to exist, and thus, even this type of exotic space travel appears to be impossible. However, there seems to be genuine evidence of novel physical phenomena, based on both new theoretical concepts as well as recent experiments that may have the potential to leading to propellantless space propulsion technology, utilizing two novel fundamental long range gravity-like fields that should be both attractive and repulsive, resulting from the interaction of electromagnetism and gravity. The current theoretical and experimental concepts pertaining to the physics of gravity-like fields are discussed together with recent experiments of producing extreme gravitomagnetic fields, performed at the Austrian Institute of Technology (AIT). The fundamental theoretical concepts termed Extended Heim Theory, EHT, are presented, and by further applying the physical ideas of $E H T$, it is argued that, in contrast to the circumferential gravity-like fields observed in the experiments at AIT, gravity-like fields acting parallel to the axis of rotation of the cryogenic disk may be producible, which should be strong enough for propulsion purposes. The basic experimental setup along with respective technical requirements as well as the resulting acceleration are described.
\end{abstract}

$\begin{array}{ll}\text { Nomenclature } \\ v_{g p}^{0} & =\text { two types of neutral gravitophotons (gravitational gauge boson) } \\ v_{g p}^{+}+v_{g p}^{-} & =\text {positive (attractive) and negative (repulsive) gravitophotons (gravitational gauge bosons) } \\ v_{g} & =\text { graviton (gravitational gauge boson, attractive) } \\ v_{q} & =\text { quintessence particle (gravitational gauge boson, repulsive) } \\ \omega_{I} & =\text { angular velocity of imaginary electrons } \\ \mathbf{B}_{G} & =\text { gravitomagnetic field vector from real moving masses }\end{array}$

\footnotetext{
${ }^{* 1}$ Senior Scientist, Institut für Grenzgebiete der Wissenschaft, 6010 Innsbruck, Austria

*2 Prof., Faculty H, Ostfalia Univ. of Applied Sciences, Campus Suderburg, 29556, Germany, Senior member AIAA.

Copyright $(2010$ by Sponsored by Ministry of Science State of Lower Saxony, Germany.. Published by the American Institute of Aeronautics and Astronautics, Inc. with permission.
} 


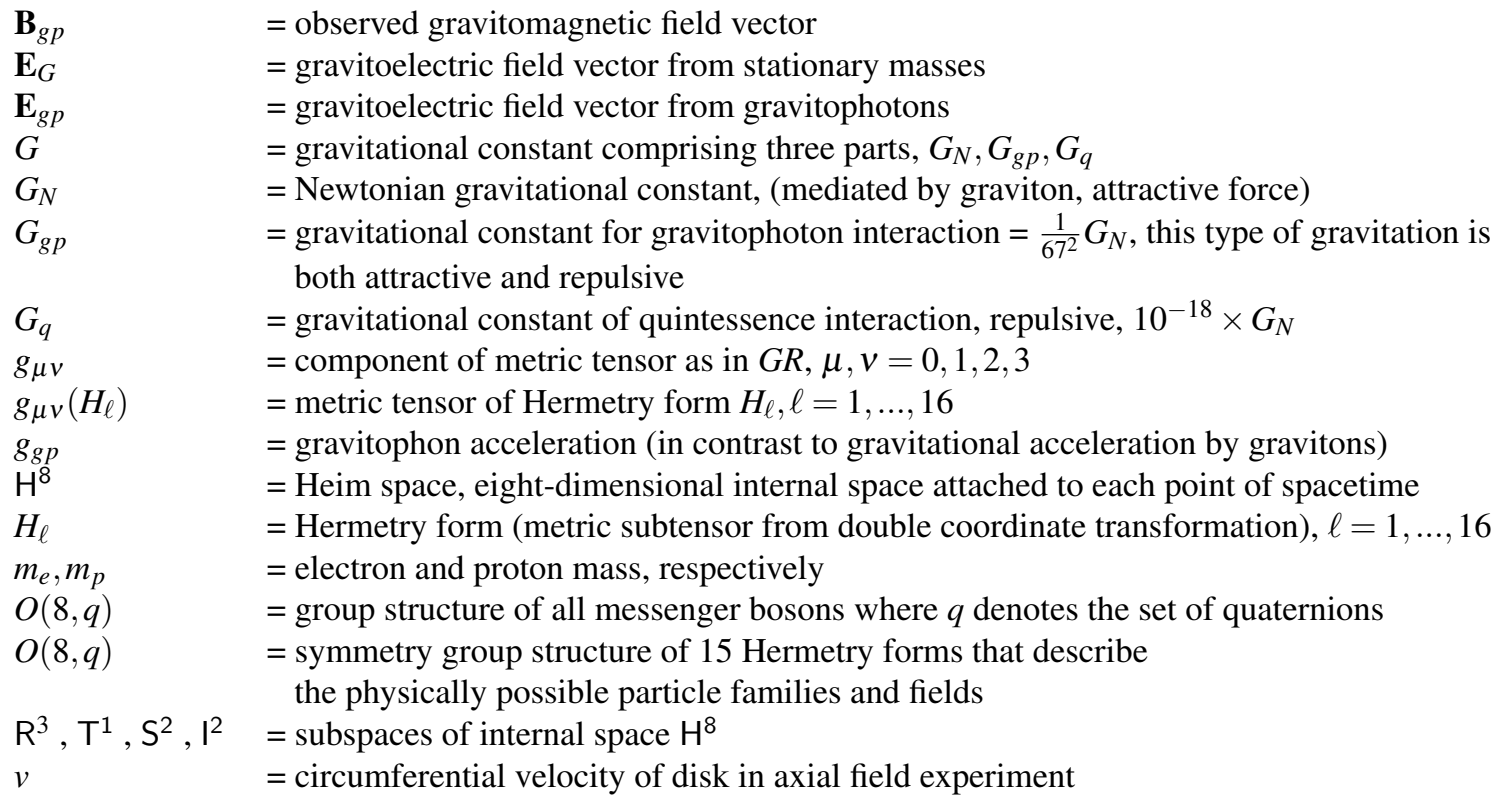

\section{Experimental and Theoretical Concepts of Novel Field Propulsion}

\section{A. Current Status of Space Propulsion}

7 HE current status of space propulsion is characterized by two contradicting scenarios. The first one, chemical 1 propulsion delivers high thrust but for several minutes only at relatively low specific impulse, and is used today to lift heavy payloads from the surface of the Earth into nearby space (for instance LEO). The second one, electric and plasmadynamic propulsion, provides low thrust over longer periods of time (up to several months) at high specific impulse, and is employed in scientific interplanetary missions of long duration. Propulsion systems can be classified according to their physical principles as thermal propulsion systems or electromagnetic propulsion systems. Advanced versions of these systems are described in the recent book by Bruno et al., ${ }^{21}$ which performs a linear extrapolation of present technology, envisaged to be realizable in 2020. Another class of advanced concepts using photonic propulsion, solar sails, or laser propulsion has been suggested. Comparing these advanced concepts with the space propulsion concepts discussed in the books by Seifert et al. (1959) ${ }^{1}$ and Corliss $(1960)^{2}$ it becomes obvious that the physical principles of all of these concepts have been around for several decades, but with regard to performance no significant progress has been made. For instance, electric propulsion systems were already tested in the 1960 s and so was nuclear propulsion. Chemical propulsion systems were never more powerful than in the 1960s.

The reason for this lack in progress is that physical laws pose strict limits on the practicality and the performance of even the most advanced propulsion systems and in practice have prevented the construction of efficient and effective propulsion systems. First, all systems considered so far operate on the basis of expulsion of mass and energy, i.e., have to obey classical momentum conservation. Hence, some kind of propellant needs to be provided. Second, the speed of light in vacuum is limited by special relativity, so interstellar travel in general does not seem to be feasible in our spacetime. This, however, is not at all a concern at present, since our current chemical propulsion systems are delivering velocities of about $10 \mathrm{~km} / \mathrm{s}$.

The state of the art of different types of advanced space propulsion concepts, based on more sophisticated physics, like space drives, warp drives, or gravity control are described in Davis and Millis (eds.) ${ }^{3}$. Nevertheless, these concepts are all utilizing one of the known four fundamental physical interactions. For instance, they are making use of special properties of the spacetime metric of general relativity $(G R)$, or try to exploit quantum entanglement for faster than light travel. Although these concepts have been known, too, in physics since the late 1930s, their engineering realization seems to be as unlikely today as it was at the time of their discovery. In particular, faster than light approaches in general relativity, GR, as investigated by Davis, Chapter 15 , in ${ }^{3}$ probably are ruled out by novel causal dynamical triangulation computer simulations ${ }^{4-6}$, since realistic spacetime topologies do not seem to allow this kind of traversable wormholes, and this type of interstellar travel thus appears unfeasible. 


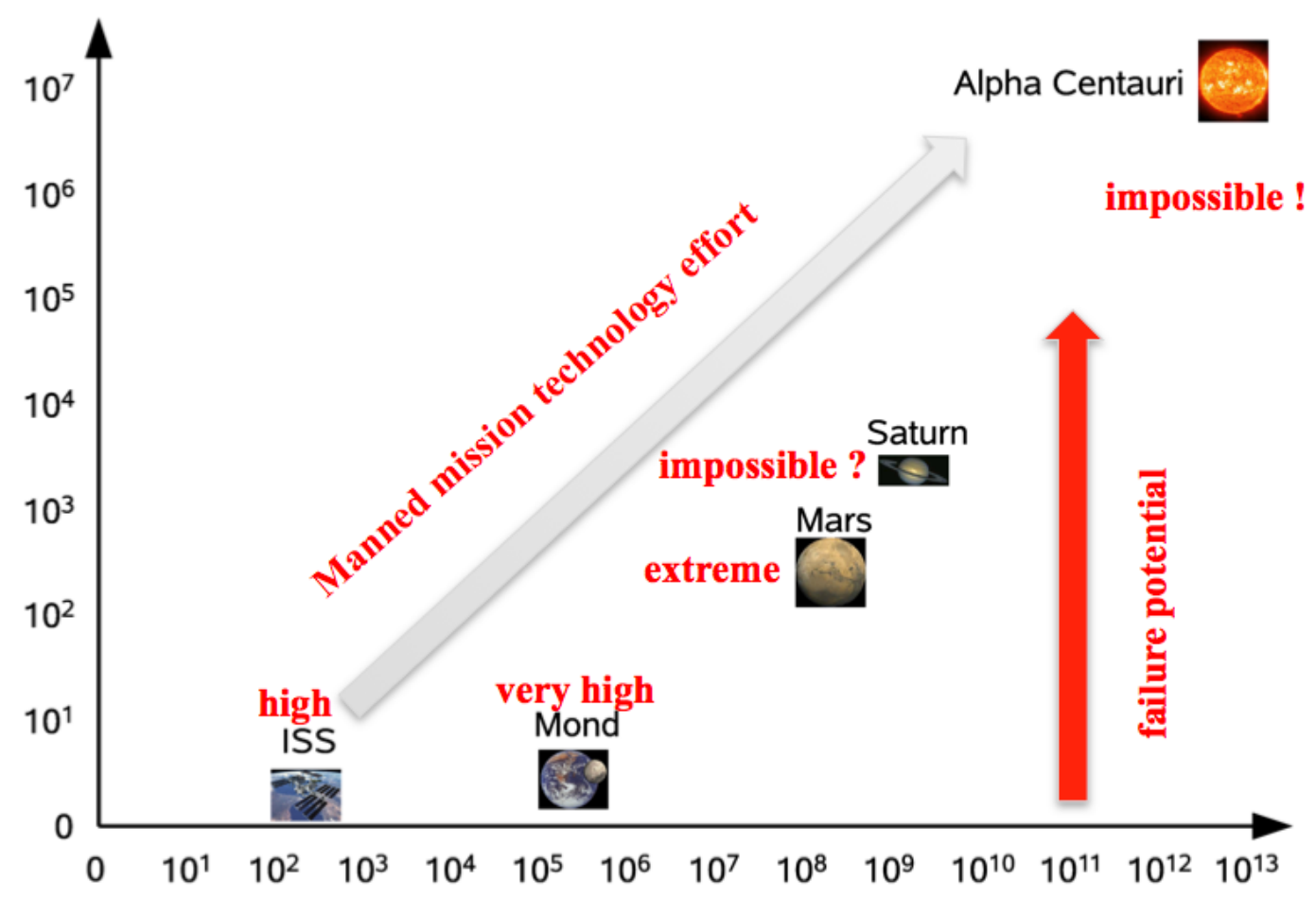

Figure 1. The picture shows the rapidly increasing mission difficulty and hazard of current space propulsio technology with respect to flight distance. The abscissa depicts distance in $\mathbf{~} \mathbf{m}$ while the ordinate shows the travel time in days. Space flight as envisaged by von Braun cannot be achieved within the stringent limits imposed by the currently four known fundamental physical interactions.

\section{B. Novel Physical Interactions and Particles}

On the other hand, current physics has no explanation for the existence of exactly four fundamental forces that is, there is a belief only about the existence of four fundamental interactions $\mathrm{s}^{7,8}$. The question therefore arises, are there any additional fundamental physical interactions? Perhaps it is classical physics and not quantum mechanics that is incomplete, i.e., there might exist additional long range interactions. This question was discussed and analyzed in more detail in several recent papers, for instance ${ }^{9,14-16}$. For several years, novel physical ideas have been presented under the name Extended Heim Theory, (EHT) ఏ by complementing spacetime with an internal eight-dimensional space $\mathrm{H}^{8}$. No extra spatial dimensions exist. that is, spacetime is $3+1$. Although these physical concepts of $E H T$ were laid out in previous publications, see for instance, ${ }^{9,14-16}$ a concise presentation for the sake of understanding of the essential features will be given in this paper. Most important, $E H T$ is postulating the existence of six fundamental forces. According to $E H T$, there should be three gravitational forces in combination with the known electromagnetic, weak, and strong interactions. Beside Newtonian gravitation (graviton $v_{g}$ attractive, coupling constant $G_{N}$ ), EHT requires the existence of two additional gravitational fields, termed gravitophoton interaction $v_{g p}$ both attractive and repulsive, which results from the conversion of electromagnetic energy into gravitational energy, and quintessence $v_{q}$, (repulsive) $^{9,10,15,16,48}$.

The question naturally arises about the physical relevance of theses ideas. Are there any, hitherto unknown, physical phenomena that might justify the assumption of the existence of additional physical interactions? The answer seems to be affirmative. In March 2006, the European Space Agency (ESA), on their webpage, announced credible experimental results, reporting on the generation of both extreme gravitomagnetic (termed frame dragging in $G R$, which, however, is too small to be measured in a laboratory on Earth) and gravity-like or gravitoelectric fields, which

\footnotetext{
${ }^{a}$ It should be noted that this doe not mean that EHT has reached the status of physical theory. At present it is a collection of novel additional physical concepts, leading to the construction of a poly-metric tensor that possibly encompasses all physical interactions ${ }^{9,14,15}$. It is a phenomenological approach to geometrize all physical interactions as envisaged by Einstein in one of his latest papers, see ${ }^{20}$. EHT constitutes a phenomenological approach to classify physical interactions and physical particles, extending the idea of mono-metric from $G R$ to poly-metric as presented by Heim ${ }^{22}$ in 1952, and Finzi ${ }^{32}$ in 1955.
} 
are acceleration fields ${ }^{b}$ performed at AIT Seibersdorf, Austria. Since then further experimental results have been published by Tajmar et al. at AIT $^{34-36}$. In July 2007, Graham et al. published a paper on the generation of a gravitomagnetic field produced by a cryogenic lead $(\mathrm{Pb})$ disk, but using a completely different measurement technique that excludes any potential perturbation by mechanical vibrations, ${ }^{37}$ see also Table $4 \mathrm{in}^{48}$. However, their results are not conclusive, since the sensitivity of their ring laser was about two orders of magnitude lower than the gyro employed at AIT. In addition, in 2008 Tajmar et al. ${ }^{38}$ published a more comprehensive set of gravitomagnetic experiments. Furthermore, in 2007 and at the end of 2008 the final results of the NASA Stanford Gravity-Probe B (GP-B) experiment ${ }^{19}$ became available. In a recent paper, $E H T$ was used to model the large unexpected gyro anomaly seen when this experiment was in orbit as well as the unforeseen acceleration and deceleration of the two gyro pairs, for details see $^{9}$.

\section{Extreme Gravitomagnetic and Gravity-Like Fields}

$G R$ predicts that any rotating massive body (Earth) drags its local spacetime around, called the frame dragging effect, generating the so-called gravitomagnetic field. This effect, predicted by Lense and Thirring in 1918, however, is far too small to be seen in a laboratory on Earth. For this reason the Gravity-Probe B (GP-B) experiment was finally launched in 2004 after more than 40 years of preparation. In this experiment the mass of the Earth was used as a test body and measuring time was about 10 months. Measurements proved to be extremely difficult. The results are presented in the final report ${ }^{19}$. On the other hand, the values measured by Tajmar et al., utilizing a small cryogenic rotating niobium $(\mathrm{Nb})$ ring, are about 18 orders of magnitude higher than predicted by $G R$, and therefore are outside $G R$. They cannot be explained by the classical frame dragging effect of $G R$, and would represent a new kind of physical phenomenon. In other words, the cryogenic $\mathbf{~ N b}$ ring in the laboratory of a mass of about 400 grams is causing approximately the same gravitomagnetic effect as a white dwarf ${ }^{9}$. In this context, it is highly interesting to compare this scenario with the so called dipole gravitational field generator, first conceived by $R$. Forward, and recently described by Davis, see Chapter 4 of $^{3}$. Instead of an electric current, Forward used a mass flow together with the Lense-Thirring effect, to produce a gravitomagnetic field $\mathbf{B}_{g}$. He showed that the mass of a white dwarf needs to be rotated to obtain an appreciable effect. From an engineering standpoint, his concept it totally unrealistic. However, compared with the recent experimental results of Tajmar, a Tajmar-Forward dipole gravitational field generator would invalidate these conclusions. Therefore, if the experiments by Tajmar et al. are correct, their physical roots must be outside GR, and thus would support the prediction of EHT about the existence of additional long range force fields. In these experiments there seems to occur an effect that increases the gravitational permeability of the vacuum, $16 \pi G_{g} / c^{2}$, by many orders of magnitude. The control parameter is temperature $T$, and a phase transition seems to occur at a certain, material dependent, critical temperature $T_{C}$, not necessarily identical with the formation of Cooper pairs, which are formed at the onset of superconductivity.

When analyzing the experiments by Tajmar et al., it became clear that though the gravitomagnetic $\mathbf{B}_{g p}$ and gravitoelectric $\mathbf{E}_{g p}$ fields (the subscript $g p$ is used to indicate that gravitophotons are deemed responsible for these extreme gravitomagnetic fields) are huge compared to the effects predicted by $G R$, they are quite small when compared to the forces needed for a space propulsion system. Also, since the gravitoelectric acceleration in Tajmar's experiments produced by the accelerated rotating cryogenic ring lies in the plane of the ring, i.e., in the circumferential direction, it cannot be directly used to accelerate a space vehicle. To this end, a force along the axis of rotation is needed. Therefore, though the AIT experiments seem to predict novel physics, and thus are of prime importance, their relevance for a gravity-engineered technology may be less pronounced. Moreover, for a space propulsion system or for the alternative generation of energy, a force acting in the axial direction without requiring the ring to be accelerated or decelerated is much more advantageous.

Since this novel effect only occurs at cryogenic temperatures, it is surmised that a phase change takes place. Moreover, EHT postulates that this phase change is leading to novel kind of particles. These virtual particles are identified as electrons of imaginary mass denoted by $e_{I}$, see Fig. 3. The coupling mechanism taking place at low temperature for these imaginary electrons is thought to lead to bosons, similar to the formation of Cooper pairs by real electrons in the superconducting phase. This process should lead to some kind of Bose-Einstein condensate, requiring, however, a conversion process that eventually is producing a force of gravitational nature.

According to $E H T$, with regard to the construction of an advanced propulsion device, a genuine base experiment should be feasible, in which the gravity-like field (acceleration field) is directed along the axis of rotation, and thus could provide the required direct mechanism for a field propulsion principle working without propellant. In addition,

\footnotetext{
${ }^{\mathrm{b}}$ In analogy to electromagnetism, gravity-like fields are denoted as gravitoelectric fields $\mathbf{E}_{G}$ since they actually produce an acceleration. One speaks of a gravitoelectric force if the $\mathbf{E}_{G}$ field is generated by stationary masses. The term gravitomagnetic force is used if $\mathbf{E}_{G}=\mathbf{v} \times \mathbf{B}_{G}$, i.e. produced by a rotating mass together with a mass density current.
} 
it is argued that the experiment can be scaled such that a propulsion system can be constructed to lift a sizable mass from the surface of the Earth. EHT will be employed to providing guidelines for the setup of this experiment and calculating the technical requirements for magnetic induction field strength, current density, and supply power. The numbers obtained should be realizable with present technology.

\section{Propellantless Space Propulsion}

Naturally, a propulsion system based on the generation of gravity-like fields, i.e., working without propellant, would be far superior over any existing propulsion technology, while its base technology might be substantially simpler and cleaner than chemical, fission, or fusion rockets. Such a system has to work in empty space and therefore, for the requirements from energy and momentum conservation, needs to interact with the spacetime field itself, i.e., any analysis based on the conservation principles has to consider the physical system formed by the space vehicle and its surrounding spacetime. This topic is discussed in more detail in Sec. III. There is, of course, insufficient knowledge at present, both theoretical and experimental, to guarantee the realization of such a device, but there is sufficient evidence both from experiment and theory to invest in the design and prototype construction of a device for generating an axial gravity-like field.

\section{Types of Matter}

The two additional gravitational fundamental forces are supposed to be mediated by gravitophotons (attractive, $v_{g p}^{+}$and repulsive, $v_{g p}^{-}$) as well as the quintessence particle (repulsive, $v_{q}$, dark energy). The quintessence particle, $v_{q}$, is assumed to be responsible for the interaction between the spacetime field (vacuum field) and ordinary matter.

Regarding Fig. 3, the outer cube, that contains non-ordinary matter (NOM), shows that there should exist the imaginary electron $e_{I}$. Comparison with the corresponding Hermetry form $H_{6}$ of charged leptons for ordinary matter $(\mathrm{OM})$ shows that subspace $\mathrm{R}^{3}$ is missing in the metric tensor of this Hermetry form, which is considered to be responsible for the existence of particles with real mass, for details see ${ }^{10} a d^{48}$. The presence of $R^{3}$ (cardinal number 3) in a Hermetry form therefore seems to be necessary for the existence of the various types of real matter. Subspace $\mathrm{T}^{1}$ (cardinal number 1) is deemed to be necessary for charged particles in conjunction with the proper Higgs field. Subspace dimensions may also play a role in computing the coupling constants (set algebra). The Hermetry form that describes neutral leptons, $e^{0}, \mu^{0}, \tau^{0}$, which belong to NOM, could also be of importance. Since these particles do not carry any electric charge they are not subject to electromagnetic interaction, and thus cannot decay in the same way through the weak interaction as their charged counterparts, and hence might be stable but invisible. Their masses could be close to those of the charged leptons. Thus, they could be candidates for dark matter. Their interaction with ordinary matter should be mediated through gravitophotons. However, there is also an alternative, termed imaginary matter, that we favor, and which is discussed in the following section.

\section{A. Imaginary Matter}

The concept of imaginary matter, i.e. particles that possess imaginary mass but otherwise have the same properties as their real counterparts, should be understood as if there existed a type of matter which is of immaterial character. This means, imaginary matter cannot be weighed (i.e. does not carry weight in our spacetime), but nevertheless its presence in spacetime can be felt through its interaction with ordinary matter.

At present we favor the idea that dark matter is comprised of imaginary matter, see Sec. IV] In EHT, since dark matter is believed to be composed of particles of imaginary mass, their presence should be perceptible in spacetime through gravitational interaction with ordinary matter. In this regard, dark matter should not be directly observable. It is unlikely that dark matter is comprised of WIMPS (Weakly Interacting Massive Particles) whose masses are supposed to be hundreds of $\mathrm{GeV}$, and thus have elucidated present accelerators. In $E H T$, dark matter interaction occurs through the gravitophoton field with coupling strength $G_{g p}$. The coupling between dark matter and ordinary matter should be given by $\sqrt{G_{N} G_{g p}}=1 / 67 G_{N}$.

In $E H T$, the set of Hermetry forms leads not only to six gravitational bosons, but also to three different types of photons, namely $\gamma$, responsible for electromagnetism, $\gamma_{I}$ the interaction boson among imaginary charged particles, and $\gamma_{I R}$ which conveys the interaction between charged particles of ordinary and imaginary matter. The three gravitational interactions, should be mediated by the six interaction bosons that is, two neutral gravitophotons, the attractive and repulsive gravitophotons as well as the graviton (Newtonian gravitation), and the quintessence particle (repulsive, 
dark energy), see Fig. 2. Here, we only note that in EHT the symmetry group for all messenger bosons is

$$
O(8, q)=S U(4)_{g l} \oplus S U(2)_{e m} \oplus S U(2)_{w} \oplus S U(2)_{g q} \oplus S U(2)_{g p} \oplus U(1)
$$

where $q$ denotes the set of quaternions. For the present discussion it may suffice to say that the first group on the right hand side defines the 8 gluons of the strong interaction, one gluon for imaginary masses, and six gluons that are responsible for interaction between real and imaginary baryons, the next group stands for the three types of photons, $\gamma, \gamma_{I}, \gamma_{I R}$ that is for the complete electromagnetic interaction. The next group describes the three well known vector bosons of the weak interaction. The following two groups are unknown in current physics, since the first one describes the gravitational bosons responsible for the axial field, $v_{g p}^{01}, v_{g p}^{+}$, and $v_{g p}^{-}$, while Tajmar's experiments would be given by $v_{g p}^{01}, v_{g}$, and $v_{q}$. The last group, having only one generator is responsible for the inertia of matter, i.e. it is some kind of all pervading Higgs field. However, it should be noted that EHT postulates a total of six Higgs and anti-Higgs fields. This topic will be discussed in much more detail in the forthcoming review paper ${ }^{11}$ . Particles of imaginary mass should also occur as virtual particles, which means that they are not present (do not occur) in the initial and final states of a reaction, but act in the intermediate steps. They might be conceived as a catalyst, enabling a novel physical interaction, namely the conversion of electromagnetic into gravitational fields as might have taken place in the recent gravitomagnetic experiments. This means that all observed particle masses and charges are still real, being the endproducts of a reaction. The neutral gravitophotons, $v_{g p}^{01}$ and $v_{g p}^{02}$ should decay into two different ways, Fig 2. The first one, in which the $v_{g p}^{01}$ decay results in positive and negative gravitophotons, should produce a vertical (axial) gravity-like field by inducing an imaginary electric current in the cryogenic rotating disk (at constant angular frequency) C Eventually this imaginary current, which is interacting with the imaginary vector potential from the superconducting coil below the disk, is converted into a real gravity-like field, see the description in Sec. IV] The second decay scheme, giving a graviton and a quintessence particle, is assumed to take place in the experiments by Tajmar et al., when a circumferential gravity-like field in the plane of the ring is produced by mechanically accelerating the cryogenic $\mathrm{Nb}$ ring. The time dependent angular frequency is considered to be the cause of the decay of $v_{g p}^{02}$, responsible of the strong gravity-like field measured by Tajmar et al. In any case, the extreme gravitational fields seem to be of electromagnetic origin. Furthermore, an interaction with spacetime (physical field) needs to be assumed to account for conservation of momentum and energy, Sec. III]

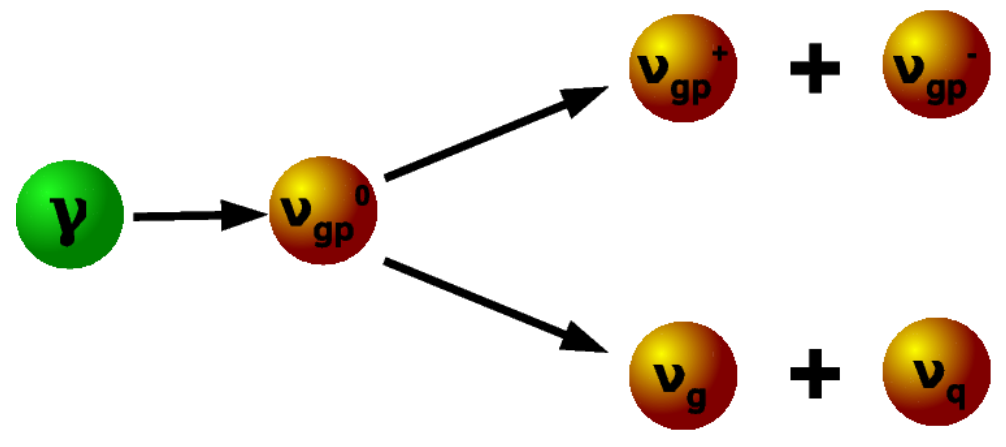

Figure 2. Hermetry form $H_{9}$ stands for the neutral gravitophoton, $v_{g p}$, produced by photon conversion, which can decay via two different channels, depending on experimental conditions. It should be noted that there are two neutral gravitophotons, denoted in the text by $v_{g p}^{01}$ and $v_{g p}^{02}$. In the picture, for the sake of simplicity, only one neutral gravitophoton is shown. The first one, upper branch, seems to take place in the generation of the axial (vertical) acceleration field, called the Heim experiment. The second branch is assumed to occur in the gravitomagnetic experiments by Tajmar et al. and Graham et al.

Moreover, there should be neutral leptons, with inertial masses of $e^{0}, \mu^{0}, \tau^{0}$ that are assumed to be close to their charged counterparts, i.e., $0.511 \mathrm{MeV} / \mathrm{c}^{2}$ for electrons, $105.66 \mathrm{MeV} / \mathrm{c}^{2}$, and $1.78 \mathrm{GeV} / \mathrm{c}^{2}$ (compared to $938 \mathrm{MeV} / \mathrm{c}^{2}$ for protons). If $e^{0}, \mu^{0}, \tau^{0}$ existed in Nature, the question naturally arises: why did not accelerators already long ago produce these particles? Accelerators or colliders produce beams of high-energy electrons or protons that are driven onto a target, or two beams are colliding from opposite directions. For some unknown reason these leptons seem not to be produced in high energy collisions. In the Standard Model, there is no place for $O M$ neutral leptons, except for the neutrinos, which, because of the experimentally established bounds on their masses, cannot contribute more than $1 \%$ to dark matter. In $E H T$, the $N O M$ counterpart to neutrinos actually are the neutral leptons $e^{0}, \mu^{0}, \tau^{0}$.

\footnotetext{
${ }^{\mathrm{c}}$ Tajmar et al. use a ring geometry, Graham et al. were using a disk, which will also be used in the proposed Heim experiment.
} 


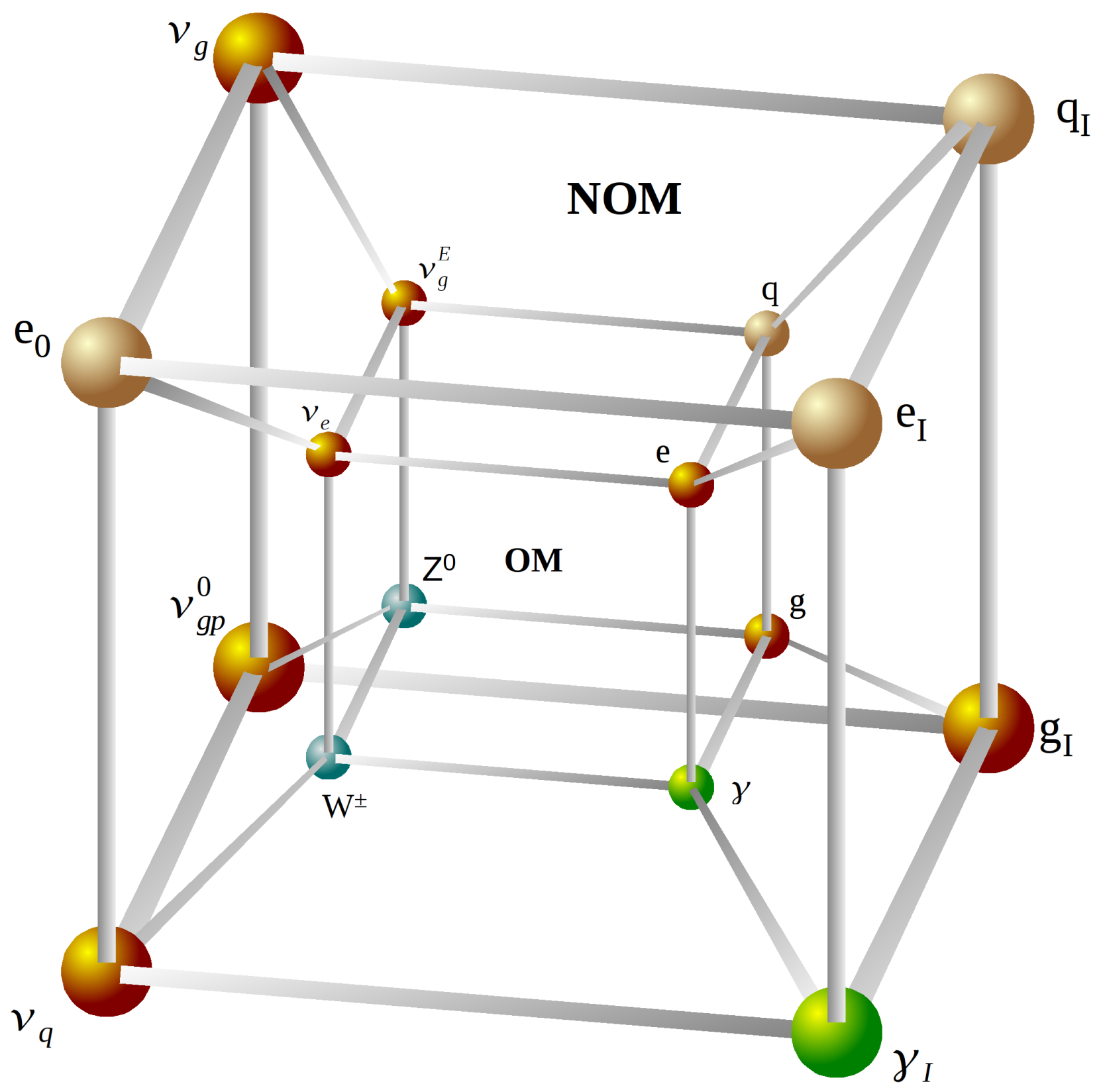

Figure 3. In $E H T$, derived from the concept of internal space $\mathrm{H}^{8}$, there exist two types of matter, ordinary matter (OM) (inner cube) and non-ordinary matter (NOM) (outer cube). In present physics, NOM does not exist. Each cube represents eight Hermetry forms. A Hermetry form stands for a family of particles, that in turn is represented by its own symmetry group. In this regard there is not a single supergroup structure that contains all forces and particles. Instead a hierarchy of groups seems to exist. The hypercube has symmetry group $O(8, q)=O(3, q)_{H} \oplus O(2, q)_{h+} \oplus O(2, q)_{h-} \oplus O(1, q)_{i n}$ where $q$ stands for the set of quaternions. The separation of $O(8, q)$ into four subgroups reflects the subspace structure of Heim space $\mathrm{H}^{8}$. Group $O(3, q)_{H}$ has 15 generators that represent 15 Hermetry forms, while Hermetry form 16 is given by group $O(1, q)_{i n}$, the inertia field that pervades the Universe. Groups $O(2, q)_{h+}$ and $O(2, q)_{h-}$ are (presently) interpreted as six Higgs and anti Higgs fields that provide mass and charge to all particles in our Universe. NOM also contains matter of imaginary type that is, dark matter is believed to be comprised of imaginary quarks, and hence should not be visible in our Universe, but its presence should be felt in our spacetime by their gravitational and electromagnetic interaction with OM, see text for further description. Thus the four-dimensional hypercube should represent all forms of matter that can impact physical events in our Universe. It should be noted that the introduction of internal space $\mathrm{H}^{8}$ along with its sub-space structure entirely fixes the structure of the symmetry groups, and thus determines the existence of particles as well as their interactions. 
In order to construct the physically meaningful set of metric sub-tensors that is, the so called Hermetry forms (the term Hermetry, coined by Heim in 1952, is referring to the physical meaning of geometry, and is a combination of the two words hermeneutics and geometry), it is postulated that coordinates of internal spaces $\mathrm{S}^{2}$ (organization coordinates) or $\mathrm{I}^{2}$ (information coordinates) must be present in any metric sub-tensor to generate a Hermetry form, whose concept was first introduced $\mathrm{in}^{22}$. From this kind of selection rule, it is straightforward to show that 12 Hermetry forms can be generated, having direct physical meaning. In addition, there are three degenerated Hermetry forms that describe partial forms occurring in $N O M$, namely the families of imaginary messenger particles, i.e. photon, gluon, and dark matter, see the outer cube of the 4D hypercube in Fig 3 . Hermetry form 16 is reserved for the inertia field, which is some kind of Higgs field pervading the whole Universe. The interpretation of the meaning of Hermetry forms was already given in Tables 1-4 of Dröscher et al. ${ }^{16}$, but has somewaht changed since then, based on new physical facts derived from the interpretation of the experiments by Tajmar et al., ${ }^{10},{ }^{48}$ and $^{14}$.

Because of the double coordinate transformation underlying the construction of the basic polymetric tensor from which all Hermetry forms are constructed, see for instance ${ }^{16,46}$, any metric tensor describing a Hermetry form is composed of a partial sum of metric terms, selected from the 64 components that comprise the complete polymetric tensor, which results from the internal space $\mathrm{H}^{8}$.

If space $\mathrm{H}^{8}$ is omitted, $E H T$ is reduced to $G R$, and only gravitation remains. It is obvious that a double coordinate transformation as employed in ${ }^{14}$ does not change, for instance, the curvature of a surface, since it is an invariant (intrinsic to the surface). However, this fact is not relevant in the construction process of the polymetric. The physical reason for the double transformation is to provide spacetime with the additional degrees of freedom, expressed by the individual components of the metric tensor from which the various Hermetry forms are constructed.

Only metric tensors representing Hermetry forms are of physical relevance, and it is clear from their construction principle that all these tensors, derived from the underlying polymetric tensor, are different. Consequently, their respective Gaussian curvatures, $K_{\ell}$, where $\ell$ denotes the index of the corresponding Hermetry form, must also be different. This is straightforward to observe, since Gaussian curvature is only a function of the first fundamental form (metric tensor components) as well as their first and second derivatives, but does not depend on the second fundamental form. Therefore, each Hermetry form $H_{\ell}$ determines its proper Gaussian curvature $K_{\ell}$, and thus curves space according to its own specific metric. Having established the qualitative physical relationship between Hermetry forms and spacetime curvature, all physical interactions are connected to spacetime curvature, similar to $G R$, and thus physics has been geometrized ${ }^{d}$. Some additional remarks between the connection of geometry and physics are in place. Internal coordinates of subspace $\mathrm{R}^{3}$ of Heim space $\mathrm{H}^{8}$ have dimension of length, and via the Compton wave length are connected to mass, and the internal coordinate of space $\mathrm{T}^{1}$ is responsible for charge. $\operatorname{In}^{16}$ it was already shown that spacetime must be quantized at about the Planck length scale (maybe the length scale is somewhat larger). Moreover, it is well known that in the case of gravitation in the Newtonian limit, metric element $g_{00}$ (time has coordinate index 0 in spacetime coordinates) is proportional to the gravitational potential equation.

\section{B. Interaction of Electromagnetism with Gravitation at Low Temperatures}

There seems to be substantial evidence of novel gravitational phenomena, based on both new theoretical concepts as well as recent experiments by Tajmar et al. at AIT, Austria that may have the potential to leading to advanced space propulsion technology, utilizing two novel fundamental force fields. According to $E H T$ these forces are represented by two additional long range gravity-like force fields that would be both attractive and repulsive, resulting from interaction of electromagnetism with gravity. At high temperature, only ordinary matter $(\mathrm{OM})$ exists. At very high temperatures, a unification of the physical interaction fields of all known forces might take place. However, at very low temperatures, a phase transition seems to occur which is generating non-ordinary matter (e.g. virtual particles of imaginary mass via the Higgs mechanism), causing an interaction between electromagnetism and gravitation. Hence, additional strong gravity-like fields may result from this conversion, being both attractive and repulsive that are coupled at these very low temperatures. It is assumed that symmetry breaking not only occurs when going from very high to lower temperatures, but also from lower temperatures to very low temperatures (starting at about $15 \mathrm{~K}$ ), and a conversion of electromagnetism into gravity-like fields may occur.

\section{Conservation Principles and the Spacetime Field}

The rocket principle requires that momentum is taken from the fuel and transferred to the space vehicle. This means the physical system to be considered for momentum conservation comprises the rocket and the ejected fuel masses.

\footnotetext{
${ }^{\mathrm{d}}$ In addition, it is assumed that color and weak charges are mediated by their Higgs bosons.
} 
The limits of this principle are too well known and cannot be overcome by technical refinement or by selecting more energetic fuel. Therefore only propellantless propulsion can be the alternative. Since conservation principles for energy and momentum must hold in all physical processes, because they represent fundamental symmetries of our spacetime, the question arises how propulsion without fuel might be physically conceivable. There seems to be only a single alternative.

According to EHT, the space vehicle is acquiring velocity by imparting an equal and opposite momentum to the spacetime field. There is also the possibility that one of the six Higgs fields pervading the Universe might be involved. Since the Higgs fields seem to confer special physical quantities (mass to hitherto massless particles, inertia, or electrical charge etc.) it is therefore assumed that interaction takes place with spacetime. Spacetime as a physical field carries momentum and energy. In case the space vehicle (or any other physical entity) is interacting with the spacetime field, the physical system to be considered for energy and momentum conservation needs to incorporate spacetime as an active partner. The momentum scale of the space vehicle is minuscule compared to that of the spacetime of the Universe, and thus the recoil kinetic energy and momentum that spacetime is receiving from the vehicle are almost negligible. Since momentum must be conserved, however, the exact momentum balance must include the momentum transferred to the spacetime field. Hence, the expansion of the Universe, at least in principle, must accelerate, although this contribution is exceedingly small and clearly cannot be measured. There may be other processes on the cosmic scale that may, however, have an observable effect. In any case, the accelerated expansion of the Universe seems to be caused by the principle of momentum conservation.

According to $E H T$, the mediators of this effect are the gravitophotons and the quintessence particle through the formation of imaginary matter. It is obvious that this principle is applicable to any other type of transportation as long as an interaction with spacetime can be established. The interpretation of the experiments by Tajmar et al. (in short Tajmar effect) by EHT leads exactly to this conclusion. Furthermore, the Heim experiment for the axial gravity-like field proposed in Sec. IV requires the existence of the gravitophotons as shown in Fig. 22 If the Tajmar effect (both, extreme gravitomagnetic field as well as circumferential gravity-like field) can be confirmed, there is some confidence that the Heim effect (axial gravity-like field) also might exist.

A simple analogy is used to differentiate between the classical rocket principle (including all other means of propulsion) and the novel field propulsion concept of EHT incorporating spacetime as a physical quantity. Suppose a boat is in the middle of a large lake or ocean. In order to set the boat in motion, a force must be mediated to the boat. The classical momentum principle requires that a person in the boat is throwing, for instance, bricks in the opposite direction to push the boat forward. However, everybody is well aware of the fact that there is a much better propulsion mechanism available. Instead of loading the boat with bricks, it is supplied with sculls, and by rowing strongly the boat can be kept moving as long as rowing continues. The important point is that the medium itself is being utilized, i.e., the water of the lake or ocean, which amounts to a completely different physical mechanism. The rower transfers a tiny amount of momentum to the medium, but the boat experiences a substantial amount of momentum to make it move. For space propulsion the medium is spacetime itself. Thus, if momentum can be transferred to spacetime by field propulsion, a repulsive or recoil force would be acting on the space vehicle moving it through the medium, like a rowing boat. The medium, spacetime, is a physical quantity, namely a field, and if properly quantized, the respective particles mediating forces should also be present. Thus, in principle, spacetime should have the capability to interact with a space vehicle. If this effect somehow can be experimentally established, the principles of momentum and energy conservation require that the combined system, i.e., both spacetime and space vehicle, are considered. According to $E H T$, this actually is the physical mechanism occurring in the experiments by Tajmar et al. and Graham et al. Important to note, this mechanism does not extract momentum from the spacetime field and transfers it to the space vehicle. Instead, an active process has to be triggered for the creation of gravitophotons, i.e., first generating a strong gravitomagnetic field, $\mathbf{B}_{g p}$. Second, in order to produce the gravity-like field seen in the experiments at AIT, experimental conditions have to be such that the $\mathbf{B}_{g p}$ field can decay, producing gravitons and quintessence particles.

The important point is that in this scheme not only gravitons exist, but also gravitophotons as well as quintessence particles. In the generation of the gravitomagnetic force via the decay of the gravitophoton, as is assumed to be the case in the gravity-like experiments by Tajmar et al., both the $O M$ (graviton, negative gravitational energy density) and NOM (quintessence particle, positive gravitational energy density) should be generated, see Figure 2 The total energy in the generation of these two particles is therefore zero. Gravitons interact with the space vehicle, i.e. they are absorbed by the space vehicle, while the quintessence particles are reabsorbed by spacetime itself. This effect causes an acceleration of the space vehicle, while the momentum of the quintessence particle is not felt by the space vehicle, but by the surrounding spacetime and leads to its expansion, because of the repulsive force, and thus total momentum is being conserved. This effect is most likely too small to be observed, but this kind of space propulsion should contribute to the expansion of the Universe. In the same way the momentum change of the ocean would not be 
discernible from the presence of a rowing boat. Perhaps a local disturbance of spacetime might be measurable in the experiments by Tajmar et al.?

In the Heim experiment (vertical gravity-like field) the neutral gravitophoton, $v_{g p}^{01}$, decays into the positive (attractive), $v_{g p^{+}}$, and negative (repulsive), $v_{g p}^{-}$, gravitophotons, Figure 2. which follows from the construction of the set of Hermetry forms that, in turn, are a direct consequence of interna Heim space and its separation into four subspaces. Again, it is assumed that the negative gravitophotons act on the spacecraft and the positive gravitophotons act on space such that total momentum is conserved. As long as the experimental conditions for the production of gravitophotons along with their respective decay are maintained, the proper acceleration field will be generated. During this period of time the interaction between space vehicle and surrounding spacetime remains. As soon as the gravitophoton production and its subsequent decay stop, the acceleration field ceases to exist.

For field propulsion to work without propellant there needs to be an active physical interaction with spacetime. The rocket principle is only concerned with the energy and momentum balance of the physical system comprising the space vehicle and its fuel. Therefore, regardless of the technology employed, this system is bound by the momentum that can be extracted from the stored fuel. Therefore this principle, by definition, cannot produce a viable propulsion system delivering high speed, long range, or high payload ratio.

An interesting question remains, namely under which conditions the production of gravitophotons on the cosmological scale can take place and how this production could have influenced the expansion of the Universe?

\section{Heim Experiment: Technology for Propellantless Space Propulsion}

Tajmar et al. were the first reporting the generation of extreme gravitomagnetic fields in the laboratory, which in $E H T$ are denoted as $\mathbf{B}_{g p}$ since they are assumed to result from gravitophotons and not from gravitons $\varsigma^{\mathrm{e}}$ It should be noted that the existence of these fields was postulated by the authors before these experiments became known, see for instance $^{45}$ that is, theory and experiments were developed independently of each other.

Modanese et al. ${ }^{18}$ have tried to explain the Tajmar effect by employing the linear Einstein-Maxwell equations, but have come to the same conclusions as the authors, namely that these equations do not even reproduce the correct sign of the gravity-like field (acceleration field) that was observed by Tajmar et al. when the angular frequency of the cryogenic ring was subject to change, i.e. the ring was accelerated. The other problem of course is that the $\mathbf{B}_{g p}$ field measured is up to 18 orders of magnitude larger than predicted by GR. Without further discussion, it should be mentioned that the often cited ratio of the gravitational and the electromagnetic force, which for proton and anti-proton is in the range of $10^{-36}$, can no longer be used to justify the negligibility of gravitational effects. This value only holds for Newtonian gravitation.

The observed gravity-like field follows a Lenz type rule, i.e., it is opposing its origin. This exhibits an electromagnetic behavior and contradicts the sign of the Einstein-Maxwell equations. The use of the non-linear equations of $G R$ cannot change this picture, since the gravitational fields observed are weak enough to fully justify the linear approximation.

The Tajmar effect cannot be explained from GR, which becomes clear in comparing the GP-B experiment with Tajmar's experiments. In GP-B, which was orbiting the Earth for more than 10 months at an altitude of about 640 $\mathrm{km}$, the predicted Lense-Thirring precession of the gyro spin axis (inertial frame dragging by the rotation of the mass of the Earth), initially pointing at a guide star (locked by a telescope), is some 42 milli-arc seconds/year. This value is small compared to the already tiny geodetic effect (spacetime curvature caused by the mass of the Earth) of 6.6 arc seconds/year. The geodetic precession occurs in the orbital plane of the satellite, while the Lense-Thirring effect causes a precession of the gyro spin axis in the same direction the Earth is rotating (the gyro is assumed to be initially in free fall along the axis of rotation of the Earth). For the GP-B experiment an inertial frame was required with nongravitational acceleration less than $10^{-13} \mathrm{~m} / \mathrm{s}^{2}$. Compared to Tajmar's equipment, his gyroscopes definitely are not capable to detect accelerations that small. One of the major challenges of the GP-B experiment was to provide such a drag-free (weightless) satellite. It is therefore impossible that Tajmar has observed any effect related to GR. His effect must therefore be outside $G R$, pointing to a new class of gravitational phenomena, provided, of course, that his measurements are correct. This is an indication that the standard picture of gravity as manifested in Einstein's $1915 G R$ does need an extension that goes beyond the picture of gravity of simply being the result of the curvature of four-dimensional spacetime. Therefore, the two additional gravitational fields as postulated in EHT, represented by gravitophotons and the quintessence particle, are at least qualitatively supported. In other words, the nature of gravity is more complex than represented by $G R$. All predictions of $G R$ are correct, but it seems that it is $G R$ which is not complete instead of $\mathbf{Q M}$ (quantum mechanics). Moreover, the geodetic and Lense-Thirring effects show that an

${ }^{\text {e}}$ The gravitons from $G R$ will not result in a gravitomagnetic field measurable in the laboratory. 
interaction between spacetime and massive bodies exist. This whould mean that the Tajmar effect, being many orders of magnitude larger, should have a much stronger interaction with its surrounding spacetime. This is exactly what is needed for propellantless propulsion, which can only work if there is an intense exchange of energy and momentum among space vehicle and spacetime, see the discussion in Sec. [III.

In order to explain the Tajmar effect, an additional assumption has to be made in order to characterize the phase transition that obviously seems to accompany all extreme gravitomagnetic phenomena. As known from superconductivity the heuristic London equations, representing the material equations, in combination with the Maxwell equations are essential to calculate both the qualitative and quantitative aspects of superconductivity in a heuristic way.

Therefore, from a physical point of view it is clear that the Einstein-Maxwell equations alone cannot describe the gravitomagnetic experiments of Tajmar, in the same way the Maxwell equations cannot account for the phenomenon of superconductivity.

- The magnitude of the extreme gravitomagnetic field points to an electromagnetic origin, being the only other long range field with sufficient coupling strength.

- Again, the London equations will be employed. Moreover, there should be a physical mechanism that converts an electromagnetic into a gravitomagnetic (or gravity-like) field.

- Such a mechanism is not conceivable within the framework of the four fundamental interactions which cannot incorporate additional gravitational fields along with their additional interaction bosons. The standard model cannot accommodate these additional particles and thus needs to be extended.

- For energy and momentum to be conserved, the interaction of the matter of the rotating disk (ring) with the surrounding spacetime field must be accounted for.

- In the Tajmar experiments the gravity-like field of the accelerated ring is acting in the plane of the rotating ring, opposing its origin. In the proposed Heim experiment, the gravity-like field of the disk, rotating at constant angular velocity, is calculated to be directed along the axis of rotation. Therefore, these two experiments seem to be based on two different physical mechanisms as depicted in Fig. 2.

- The first neutral gravitophoton, indicated by $v_{g p}^{01}$, which is deemed to be responsible for the Heim effect, should decay into the positive (attractive) $v_{g p}^{+}$and negative (repulsive) $v_{g p}^{-}$. The resulting gravity-like field is pointing in axial direction.

- The second neutral gravitophoton, indicated by $v_{g p}^{02}$ decays only if the ring is being accelerated, and the resulting gravity-like field is in circumferential direction, and thus this decay route is believed to occur in the experiments by Tajmar et al., denoted as Tajmar effect.

- From a technological point of view the axial gravity-like field is the one that could provide the enabling technologies for propellantless propulsion and novel air and land transportation systems as well as green energy generation etc. At present, it does not seem possible to fully assess the technological consequences of the existence of such a field.

- The two experiments for circumferential and axial gravity-like fields are fundamentally different, but in both cases a conversion from electromagnetic to gravitational fields seems to take place, triggered by the generation of imaginary electrons, see NOM cube in the 4D hypercube of Fig. 3

In the framework of the current paper a full discussion of the implications of imaginary matter cannot be given, but the basic facts of the conversion mechanism will be presented. It seems that not only particles and their anti-particles, but, under certain conditions, also particles and their ghost or shadow particles (i.e. imaginary mass) particles exist, or, at least, can be created under special experimental conditions. At temperatures low enough for the respective phase transition to occur, it seems that the imaginary electrons being produced are forming bosons comprising six imaginary electrons $e_{I}$. The imaginary current due to these sextets is deemed to result in an imaginary vector potential $\mathbf{A}_{I}$ whose interaction with the imaginary quarks $q_{I}$ (protons) in the rotating disk is eventually leading to a real physical interaction which appears in the form of gravity-like fields. The physical mechanism is complex, but, as can be seen from the experimental setup of Tajmar et al., the generation of the circumferential gravity-like field is surprisingly simple. The same should hold true for the axial gravity-like field experiment. Any propellantless space propulsion technology therefore would be substantially simpler and efficient than currently used chemical propulsion, and also inherently safer as well as far more economical. 
The experiment for the axial field comprises a cryogenic disk comprised of a given material, denoted as $M_{D}$ having a diameter of about $0.2 \mathrm{~m}$, rotating at circumferential velocity $v$. Below the disk a superconducting coil is placed, made of material $M_{C}$, that comprises $N$ turns. The disk may also reside inside the coil. It should be noted that disk and coil material need to be complementary. In the experiments by Tajmar et al. a $\mathrm{Nb}$ ring and an $\mathrm{Al}$ sample holder seem to give the best results. The third part is a simple device to ensure that the current of imaginary electrons is coupled into the coil. In order to achieve this the wire of the coil is cut through and a non-superconducting disk of about $1 \mathrm{~mm}$ thickness is introduced. The Cooper pairs cannot tunnel through this layer, since its thickness far exceeds the $10 \AA$ of the Josephson effect. However, the Compton wave length of the imaginary electrons is much larger, because of the small limit velocity $c_{I}$ in solids for particles of imaginary mass, and thus the $e_{I}$ should be capable of tunneling through, leading to the imaginary current $I_{I}$ that gives rise to the imaginary vector potential $\mathbf{A}_{I} \mathrm{f}^{[\mathrm{f}}$

In the following, the main steps for calculating the axial field strength are presented. In electrodynamics, given a current distribution with current density (moving charges) $\mathbf{j}(\mathbf{x})$, the force on this current distribution due to the magnetic induction $\mathbf{B}$ is

$$
\mathbf{F}=\int \mathbf{j}(\mathbf{x}) \times \mathbf{B}(\mathbf{x}) d^{3} x
$$

In $G R$ the same formula holds for calculating the gravitomagnetic Lorentz force, replacing the current density by the mass flux density $\mathbf{j}(\mathbf{x})=\rho_{m} \mathbf{v}$ where $\rho_{m}$ is mass density, $\mathbf{v}(\mathbf{x})$ velocity of the moving mass, and $\mathbf{B}_{g}$ now denotes the gravitomagnetic field as derived from $G R$. It should be noted that sometimes the vector $\mathbf{r}$ instead of $\mathbf{x}$ is used. In any case $d^{3} x$ indicates the three-dimensional volume element independent of the coordinate system chosen. The electromagnetic Lorentz force does not follow from the Maxwell equations, but has to be postulated from the Lagrange function specifying the coupling of an electric charge to the vector potential, i.e. $L=q \mathbf{v} \cdot \mathbf{A}$. However, in the gravitational case, the Lorentz force law follows from the geodesic equation describing the motion of a test particle in a gravitational field. From the Lageos satellites and the GP-B experiment we know this to be true for gravitomagnetic fields $\mathbf{B}_{g}$ resulting from large rotating masses, as given in Eq. (3). We assume that Eq. (2) holds for all gravitational interactions, independent of the origin of the gravitomagnetic field, i.e., it should not make any difference whether this field is produced by gravitons or gravitophotons that is, for the $\mathbf{B}_{g p}$ field.

According to $G R$, a material rotating spheroidal body of angular momentum $\mathbf{J}=\int \rho_{m} \mathbf{r} \times \mathbf{v} d^{3} x$ is generating a gravitomagnetic field $\mathbf{B}_{g}$, where $\mathbf{v}=\omega \times \mathbf{r}$ is the circumferential velocity and $d^{3} x$ denotes the volume element. The subscript $g$ indicates that this gravitomagnetic field is due to Newtonian gravitation

$$
\mathbf{B}_{g}=2 \frac{G}{c^{2}} \frac{\mathbf{J}-3\left(\mathbf{J} \cdot \hat{\mathbf{e}}_{r}\right) \hat{\mathbf{e}}_{r}}{r^{3}} .
$$

If we insert this expression into Eq. 22, the resulting force on a moving mass distribution is found. For instance, the force as well as the torque on a rotating gyro can be determined that is, because of the torque, the gyro will start to precess, or, in other words, the gyro is dragged. This can also be interpreted such that the inertial frame of the gyro is dragged, of which the gyro defines one of the axes.

However, the gravitomagnetic mechanism of $G R$ clearly is not the mechanism that occurs in the experiments by Tajmar et al. As the experiments demonstrate, the process is a solid state phenomenon, depending on a phase transition, triggered by temperature. Therefore, the generation of the gravitomagnetic field follows a totally different mechanism different than $G R$. Hence, the gravitomagnetic field denoted $\mathbf{B}_{g p}$ must be calculated by a different physical model. According to EHT,

- the gravitomagnetic field $\mathbf{B}_{g p}$ is generated by new types of bosons, termed gravitophotons $v_{g p}^{ \pm}$,

- the origin of the $\mathbf{B}_{g p}$ is the electromagnetic field,

- conversion from electromagnetism to gravitomagnetism seems to follow the reaction chain $\gamma \rightarrow \gamma_{I R} \rightarrow \gamma_{I} \rightarrow$ $v_{g p}^{01} \rightarrow v_{g p}^{+}+v_{g p \rightarrow v_{g}+v_{q}}^{-}$,

- the $v_{g}$ gravitophoton confers the momentum to the space vehicle, the $v_{q}$ gravitophoton provides negative momentum to the surrounding spacetime which therefore expands, the total momentum of the physical system remains unchanged, i.e. zero.

As additional material equations for gravitophoton interaction, in analogy to superconductivity, the London equations are employed in determining the magnitude of the $\mathbf{B}_{g p}$ field in conjunction with the conversion mechanism, i.e.

\footnotetext{
${ }^{\mathrm{f}}$ It is not known if the direct imaginary current is superimposed by a high frequency alternating imaginary current as observed for Cooper pairs in the Josephson effect.
} 
its magnitude is determined by the underlying physics of the conversion process. It is well known that in the superconducting case a real super current is generated by electron Cooper pairs, formed by a phase transition at critical temperature $T_{C}$, described by the heuristic London equation

$$
\mathbf{B}=-\frac{2 m_{e}}{e} \omega
$$

where $\mathbf{B}$ is the magnetic induction field caused by the Cooper pairs. In the experiments by Tajmar et al., as discussed in Sec. I, an extreme gravitomagnetic field is generated. For the explanation of these experimental results as well as for the Heim experiment, it is assumed that the current of the superconducting electrons (Cooper pairs) causes a current of imaginary electrons. Imaginary particles are formed via the Higgs mechanism, for instance, as described by M. Kaku, Chap. $10^{23}$, further details are also given $\mathrm{in}^{48}$. Due to the interaction of the imaginary particles with OM, they should not behave like tachyons. For the Heim experiment, the imaginary current needs to be coupled into the superconducting coil by some kind of tunnel effect as stated above. The Cooper pair current is not important by itself, it only acts as the source for the accompanying imaginary current

$$
\mathbf{B}_{e_{I}}=-i \sqrt{\alpha_{I}} \frac{6 m_{e}}{e} \omega_{I}
$$

where $\omega_{I}$ denotes the angular frequency of the imaginary bosons formed by the coupling of the $e_{I}$. Finally the conversion of the imaginary mass $m_{e_{I}}$ into real mass $m_{e}$ takes place g via the conversion from electromagnetic into gravitational fields. Therefore, the real values are already used in the above equation. It is important to ensure experimentally that an imaginary current is flowing in the coil, i.e. an experimental mechanism must be provided to couple this current into the coil, once the real super-current sets in. It should be mentioned that the chain of formation of the three types of photons $\gamma \rightarrow \gamma_{I} \rightarrow v_{g p}^{01}$ takes only place below a certain critical temperature.

The value of the coupling constant $\alpha_{I} \approx \frac{1}{228}$ is related to the radiation correction of the Higgs field, see M. Kaku ${ }^{23}$ p.353, via $\sqrt{\lambda} \approx \alpha_{I}$. The factor $6 m_{e I}$ is obtained when an imaginary instead of a real mass is considered.

The total Lagrange density for the conversion from electromagnetism to gravitation is assumed to be $\mathcal{L}=0$ that is,

$$
i \alpha_{I} e^{+} \mathbf{v} \cdot \mathbf{A}_{e_{I}}+m_{p} \mathbf{v} \cdot \mathbf{A}_{g p}=0
$$

where $\mathbf{v}$ is the velocity of the rotating disk above the coil. The decay of the neutral gravitophoton of the first type, $v_{g p}^{01}$, into $v_{g p}^{01} \rightarrow v_{g p}^{+}+v_{g p}^{-}$leads to the real gravitophoton potential $\mathbf{A}_{g p}$. It is assumed that the conversion of the imaginary magnetic induction field $\mathbf{B}_{I}$ into the gravitomagnetic field $\mathbf{B}_{g p}$ follows from the transformation equation of the electromagnetic field, which is a Lorentz transformation. Lorentz transformations not only act on spacetime but also on the internal spin states, resulting in their mixing. ${ }^{24}$ The same is supposed to be true for the mixing of Hermetry forms in internal space $\mathrm{H}^{8}$. Thus the resulting field is

$$
\mathbf{B}_{g p} \sim \mathbf{v} \times \mathbf{B}_{e_{I}}
$$

which means that, if the $\mathbf{B}_{e_{I}}$ of the London equation is directed along the $z$-axis, the resulting $\mathbf{B}_{g p}$ is pointing in $\hat{\mathbf{e}}_{r}$ direction, if cylindrical coordinates are used. It should be noted that $\mathbf{A}_{e_{I}}$ is the imaginary vector potential that belongs to Eq. 5. Thus the resulting gravitomagnetic field is

$$
\mathbf{B}_{g p}^{+}=\alpha_{I} \frac{6 m_{e}}{m_{p}} \omega_{I} \hat{\mathbf{e}}_{r} .
$$

As mentioned above, the Lorentz equation also holds for the gravitophoton force (it should be noted that the Maxwell equations and the Einstein-Maxwell equations are similar, and the fully nonlinear equations Einstein field equations are only of interest in the direct neighborhood of black holes or for distances comparable to the diameter of the Universe) that is, the gravity-like force is given by

$$
\mathbf{F}=m \mathbf{v} \times \mathbf{B}_{g p} \sim m \mathbf{v} \times\left(\mathbf{v} \times \mathbf{B}_{e_{I}}\right),
$$

which means that the resulting force is in the direction of the $\mathbf{B}_{e_{I}}$ field, which, in the Heim experiment is the axial direction. The gravitophoton $g_{g p}^{+}$acceleration then has the form

${ }^{\mathrm{g}}$ The charge of the imaginary electron $e_{I}$ and electron $e$ are the same. 


$$
g_{g p}^{+}=\alpha_{I} \frac{6 m_{e}}{m_{p}} \frac{v^{2}}{c} \omega_{I}
$$

Since $\mathbf{v}$ is the circumferential speed of the rotating disk, the average velocity of the particles in the disk is given by

$$
v_{A}^{2}=\frac{1}{3} v^{2}
$$

and therefore the axial acceleration is

$$
g_{g p}^{+}=\alpha_{I} \frac{2 m_{e}}{m_{p}} \frac{v^{2}}{c} \omega_{I}
$$

Since finally the gravitophoton acceleration field is converted into a graviton field, the ratio of the gravitational coupling factors $G_{N} \times G_{g p}^{-1}=67$ is introduced. The new variables appearing in the following equation are specified in the numerical example below.

$$
g_{g}=\sqrt{\frac{G_{N}}{G_{g p}}} \alpha_{I} \frac{2 m_{e}}{m_{p}} \frac{\rho_{D} A_{D}}{\rho_{0 D} A_{0 D}} N\left(\frac{A_{C}}{A_{0 C}}\right) \frac{v^{2}}{c} \omega_{I}
$$

It should be mentioned that the real super-current in the coil is limited according to Eq. 14

$$
I_{L}=\frac{2 \pi R}{\mu_{0}}\left(\frac{A_{C}}{A_{0 C}}\right) \frac{6_{m_{e}}}{e} \omega_{I}
$$

where $R$ is the radius of the coil. Only for a real current $I<I_{L}$ imaginary electrons can assume part of the wire cross section of the coil.

As an example for a laboratory experiment to producing a sizable axial field a disk of $d=0.2 \mathrm{~m}$ diameter together with the following parameters is used: $\frac{m_{e}}{m_{p}}=\frac{1}{1836}, \frac{\rho_{D}}{\rho_{0 D}}=0.19, \frac{A_{D}}{A_{0 D}}=\frac{10^{3}}{36}$, where $\rho_{0 D}$ and $A_{0 D}$ are reference density and reference area of the disk, and $N=50$ is the number of turns of the coil. A value of $A_{C} / A_{0 C}=5$ is chosen, where $A_{C}$ and $A_{0 C}$ are the cross section and the so called reference cross section of the coil, respectively. The circumferential speed of the disk is $v=50 \mathrm{~m} \mathrm{~s}^{-1}$ and $\omega_{I}=10^{5} \mathrm{~s}^{-1}$. Inserting these values results in

$$
g_{g}=67 \times \frac{1}{228} \times \frac{2}{1836} \times 0.19 \times \frac{10^{3}}{36} \times 50 \times 5 \times \frac{2500}{3 \times 10^{-8}} \times 10^{5} \times \frac{1}{9.81} g=\mathbf{3 . 6} \times \mathbf{1 0}^{-\mathbf{2}} g
$$

where $g$ denotes the acceleration of the Earth. For the limit of the real current $I_{L}$ one finds

$$
I<I_{L}=\frac{2 \pi R}{\mu_{0}}\left(\frac{A_{C}}{A_{0 C}}\right) \frac{6 m_{e}}{e} \omega_{I} \approx 10 A .
$$

\section{Conclusions and Future Activities}

Since 2002 ideas of a geometric approach (termed Extended Heim Theory $(E H T)$ ) were published by the authors with the aim to determine all possible physical interactions and to associate to each interaction its proper metric tensor. This goal is achieved by complementing four-dimensional spacetime with an internal eight-dimensional space $\mathrm{H}^{8}$ comprising a set of four subspaces. It turned out that this approach, which differs form the introduction of extra spatial dimensions, predicts six fundamental physical interactions, namely three types of gravitational fields, electromagnetism as well as the weak and strong interactions ${ }^{9,15,16}$. In EHT gravitation can be both attractive and repulsive. $E H T$ also predicts the existence of virtual particles of imaginary mass, responsible for the conversion of electromagnetic into gravitational fields. There should be also three different types of photons. Imaginary particles might be accountable for the invisible dark matter, recognizable only through its gravitational interaction with ordinary matter. Charged ordinary and imaginary matter can also interact electromagnetically, because of the three different types of photons postulated in EHT: $\gamma$ which is the ordinary photon, $\gamma_{I}$ responsible for the interaction between charged imaginary particles, and $\gamma_{I R}$ that conveys the interaction between charged imaginary and real particles. Concerning the speed of gravitational interactions, $E H T$, has determined three widely different propagation speeds.

- The interaction of Newtonian gravity (force) is almost instantaneous, that is $c_{g}=2.5 \times 10^{10} c$, 
- the propagation speed of gravitational waves (energy) and neutral gravitophotons is $c$ (speed of light in vacuum),

- the quintessence interaction, i.e. the repulsive part of gravitation, (acceleration of the Universe propagates at a very low speed $c_{I}$ with $c^{2}=c_{g} c_{I}$, and thus imaginary particles can form at very low velocities. The velocity $c_{I}$ is also the propagation speed of imaginary particles in matter.

- To all three propagation speeds their proper Lorentz transformation is assigned.

Numerous experiments by Tajmar et al. at AIT Seibersdorf carried out since 2003, and first published in 2006, report on the laboratory generation of extreme gravitomagnetic as well as gravity-like fields, and thus seem to corroborate the theoretical findings of EHT. The gravitomagnetic effects measured are about 18 orders of magnitude larger than predicted by the so called Lense-Thirring effect of GR. In other words, the rotating niobium ring utilized by Tajmar et al., having a mass of some 400 grams, produces a frame dragging effect similar to the mass of a white dwarf ${ }^{9}$. These experiments were repeated by Graham et al. ${ }^{37}$ in 2007 , and more recently by Tajmar et al. ${ }^{36}$ including a comparison between the two experiments.

If the experiments of Tajmar and Graham are correct, a similar effect should have been observed in the NASAStanford Gravity-Probe B experiment as calculated in $^{9}$. Indeed, a large gyro anomaly was observed in GP-B, but attributed to an electrostatic patch effect. In how far there is room for the extreme gravitomagnetic effect, as predicted by $E H T$ therefore is an open question. $E H T$ was used to analyze all of these experiments, and also (approximately) predicted the magnitude of the gyro misalignment in the GP-B experiment resulting from the postulated extreme gravitomagnetic spin-spin interaction. The in orbit observed gyro misalignment was attributed to the generation of gravity-like fields acting between the cryogenic $\mathrm{Nb}$ coated gyros (cf. Tajmar experiments) in each of the two gyro pairs. The two counter-rotating gyro pairs utilized in the GB-P experiment exhibited asymmetric misalignment, depending on the direction of their rotation. Theoretical predictions by EHT and measured misalignment were compared with the in orbit measurements and gave reasonably good agreement. Hence, it remains to be seen whether the electrostatic patch effect, used in the post-flight analysis to predict gyro misalignment by the Stanford team, is capable to completely accounting of both the magnitude and the type of anomaly observed. According to EHT, this anomaly should not be totally explainable by classical effects, i.e. electrostatic forces etc. The result of GP-B is that the Lense-Thirring (frame dragging) effect exists exactly as predicted by $G R$ though the initially targeted accuracy of $1 \%$ could not not be obtained. Therefore, there is no room using a modification of the Lense-Thirring effect to explaining the huge observed gravitomagnetic fields. The calculations done in ${ }^{9}$ fall into the range of the observed gyro misalignment, but it can only be surmised that there is room for the extreme gravitomagnetic effect by $E H T$.

In summary, the present situation is characterized by the fact that numerous gravitational experiments have been performed over a period of four years, employing different measurement techniques, showing similar, but unexpected and unexplainable results if the framework of $G R$ is employed. Measurement techniques in all experiments are clearly state of the art, in particular for the GP-B experiment.

In all experiments a phase transition seems to have occurred at low temperature (not necessarily at $T_{C}$, the critical temperature for superconducting), and possibly boson interaction took place that is, formation of virtual bosonic imaginary particles from imaginary electrons $e_{I}$. GR cannot be used to explain these phenomena, even if the full nonlinear Einstein field equations were used. The Lageos satellites and the GP-B experiment have clearly demonstrated that the inertial frame dragging effect, even from celestial bodies, is extremely small and within $G R$. These facts provide evidence for entirely novel physics in the form of additional long range fundamental forces. In this regard, $\boldsymbol{G R}$ seems to be incomplete and not $\boldsymbol{Q M}$, something Einstein would have been shocked to learn about.

At present, there are qualitative but also substantial quantitative uncertainties concerning the theory of gravitomagnetic physics. The unusual, laboratory generated extreme gravitomagnetic and gravity-like fields point to a novel mechanism and, as predicted by EHT, may be the result of two additional gravity-like fields in combination with non-ordinary matter, i.e. imaginary matter. The experiment for generating an axial field, termed Heim experiment, is surprisingly simple and would not require excessive magnetic fields or currents, except that liquid He is necessary to achieve the postulated phase transition. The Heim experiment can therefore be performed with current technology and, if successful, would allow to engineer gravity in a way similar to electromagnetism. Of great practical importance would also be the aspect of energy conversion from the direct interaction between electromagnetism and gravitation, or from employing gravity-like fields as plasma stabilizers in nuclear fusion, for instance, in simple configurations like magnetic mirrors.

The gravity-like fields would also lead to entirely novel technologies in the area of transportation be it on the ground and on water, or in the air. In addition, these fields might also be usable in energy generation leading to energy research that might be highly relevant for the near future. 
The theoretical work to be performed needs to focus on both the fundamental aspects of the symmetry groups as well as on determining the technical and experimental details in order to experimentally realize the proposed axial field. Naturally, many theoretical questions remain open concerning the six fundamental forces as well as in particular the role and the interaction scenarios of the postulated imaginary matter.

Most important, spacetime is supposed to have an active role being part of the physical system that means, propellantless propulsion would interact with the spacetime field exchanging energy and momentum. Such a space propulsion system would in principle lead to a minuscule acceleration of the Universe, though in practice this acceleration would hardly be noticeable, in the same way the momentum change of a rowing boat, impeded by its sculls to the total momentum of the water of the ocean, would remain undetectable.

\section{Acknowledgment}

This paper is dedicated to Dr. William Berry (ret.), head of Aerothermodynamics and Propulsion Division, ESAESTEC, Noordwijk, The Netherlands on the occasion of his 75th birthday.

The assistance by M.Sc. O. Rybatzki, Faculty Karl-Scharfenberg, Univ. of Applied Sciences, Salzgitter Campus in preparing the figures is gratefully acknowledged.

The authors are grateful to Prof. Dr. M. Tajmar, KAIST Seoul, Korea for providing measured data as well as for numerous comments regarding comparisons between $E H T$ and the gravitomagnetic experiments.

The authors are most grateful to Prof. P. Dr. Dr. A. Resch, director of the Institut für Grenzgebiete der Wissenschaft (IGW), Innsbruck, Austria for his support in writing this paper.

The second author is indebted to his colleague Prof. Thomas Waldeer, Ostfalia Univ., Suderburg for proofreading the paper and suggesting improvements.

\section{References}

${ }^{1}$ Seifert, H. (ed.).: Space Technology, Wiley 1959.

${ }^{2}$ Corliss, R. C.: Propulsion Systems for Space Flight, McGraw-Hill 1960.

${ }^{3}$ Hathway, G.D.: Gravitational Experiments with Superconductors: History and Lessons, Chap. 5, in M.G. Millis, E.W. Davis (eds.) : Frontiers in Propulsion Science, American Institute of Aeronautics and Astronautics 2009, and Davis, E.W., Chaps. 4 and 15.

${ }^{4}$ Loll, R. : The Emergence of Spacetime or Quantum Gravity on Your Desktop, arXiv:0711.0273v2, 16 April 2008.

${ }^{5}$ Ambjorn,J., Jurkiewicz, J., R. Loll: The Self Organizing Quantum, Scientific American, August 2008.

${ }^{6}$ Ambjorn,J., Jurkiewicz, J., R. Loll: Quantum Gravity: the art of building spacetime, Chap. 18 in Quantum Gravity, ed. D. Oriti, Cambridge Univ. Press, 2009.

${ }^{7}$ Sarkar, U.: Particle and Astroparticle Physics, Taylor\&Francis 2008.

${ }^{8}$ Hasinger, G.: Das Schicksal des Universums, Goldmann 2009.

${ }^{9}$ Dröscher, W., J. Hauser: Gravity-Like Fields and Space Propulsion Concepts, AIAA 2008-5124, 44th AIAA/ASME/SAE/ASE, Joint Propulsion Conference \& Exhibit, Hartford, CT, 20-23 July 2008, 19 pp.

${ }^{10}$ Hauser, J., Dröscher,W., : Emerging Physics for Space Propulsion Science, Space, Propulsion \& Energy Sciences International Forum SPESIF-2010, American Institute of Physics, Conference Proceedings, 978-7354-0749-7/10, 15 pp.

${ }^{11}$ Hauser, J., W. Dröscher, W.,: Review of Emerging Physics for Novel Space Propulsion Science: Physics of Extreme Gravitomagnetic and Gravity-Like Fields, to be submitted to Journal of Progress in Aerospace, 2010.

${ }^{12}$ Hobson, M.P., G. Efstathiou, A.N. Lasenby: General Relativity, Cambridge Univ. Press, 2006.

${ }^{13}$ Smolin, L.: Atoms of Space and Time, Scientific American, January 2004.

${ }^{14}$ Dröscher,W., J. Hauser: Current Research in Gravito-Magnetic Space Propulsion, Paper O-42, 7th International Symposium on Launcher Technologies, 2-5 April 2007, Barcelona, Spain, 16 pp.

${ }^{15}$ Dröscher,W., J. Hauser: Advanced Propulsion Systems from Artificial Gravitational Fields, AIAA 2007-5595, 43th AIAA/ASME/SAE/ASE, Joint Propulsion Conference \& Exhibit, Cincinnati, OH, 8-11 July 2007, 15 pp.

${ }^{16}$ Dröscher,W., J. Hauser: Spacetime Physics and Advanced Propulsion Concepts, AIAA 2006-4608, 42nd AIAA/ASME/SAE/ASE, Joint Propulsion Conference \& Exhibit, Sacramento, CA, 9-12 July 2006, 20 pp., (available as revised extended version 20 August 2006 at www.hpcc-space.de).

${ }^{17}$ Dröscher,W., J. Hauser: Guidlines for a Space Propulsion Device, AIAA 2004-3700, 40th AIAA/ASME/SAE/ASE, Joint Propulsion Conference \& Exhibit, Fort Lauderdale, FL, 11-14 July 2004, 27 pp.

${ }^{18}$ Modanese G., oral communication, 30 May 2010 during a meeting at Institut für, Grenzgebiete der Wissenschaft, Innsbruck, Austria.

${ }^{19}$ Stanford University: Gravity Probe B Final Report, einstein.stanford.edu, December 2008. 
${ }^{20}$ Einstein, A.: On the Generalized Theory of Gravitation, Scientific American, April 1950, Vol 182, NO.4.

${ }^{21}$ Bruno, C., A.G. Accetura.: Advanced Propulsion Systems and Technologies, Today to 2020, AIAA 2008, 489 pp.

${ }^{22}$ Heim, B.: Vorschlag eines Weges einer einheitlichen Beschreibung der Elementarteilchen, Zeitschrift für Naturforschung, 32a, 1977, pp. 233-243.

${ }^{23}$ Kaku, M.: Quantum Field Theory, Oxford, 1993.

${ }^{24}$ Bergström, L., Goobar, A..: Cosmology and Particle Astrophysics, Springer, 2004, Chap.6.

${ }^{25}$ Schmüser, P.: Feynman-Graphen und Eichtheorien fúr Experimentalphysiker [Lecture Notes in Physics), Springer 1998.

${ }^{26}$ Zee, A.: Quantum Field Theory in a Nutshell, Princeton University Press, 2003.

${ }^{27}$ Lahore, A., P., B. Pal: Quantum Field Theory, 2nd Ed., Alpha Science, 2004.

${ }^{28}$ Scheck, F.: The Higgs Mechanism and Spontaneous Symmetry Breaking, Chap. 11 in Noncommutative Geometry and the Standard Model of Elementary Particle Physics, eds. F. Scheck, H. Upmeier, W. Werner, Springer 2002.

${ }^{29}$ Tajmar, M. et al.: Hypothetical Gravity Control and Possible Influence on Space Propulsion, JournalofPropulsionandPower, Vol. 21, No. 4, July-August 2005.

${ }^{30}$ Heim, B. (ed. A. Resch): Mensch und Welt, Resch Verlag Innsbruck 2008.

${ }^{31}$ Heim. B., Dröscher, W.: Strukturen der Physikalischen Welt und ihrer nichtmateriellen Seite, Resch Verlag, Innsbruck, Austria, 1996, 2nd ed. 2007.

${ }^{32}$ Cardone, F. and R. Mignani: Energy and Geometry, World Scientific 2004.

${ }^{33}$ Woods C. et al.: Gravity Modification by High Temperature Superconductors, AIAA 2001-3363, 37 th AIAA/ASME /SAE/ASE, Joint Propulsion Conference \& Exhibit, Salt Lake City, Utah, 8-11 July 2001.

${ }^{34}$ Tajmar, M. et al.: Experimental Detection of the Gravitomagnetic London Moment, http://arxiv.org/abs/gr-qc/0603033, 2006.

${ }^{35}$ Tajmar, M. et al.: Measurement of Gravitomagnetic and Acceleration Fields Around Rotating Superconductors, STAIF AIP, February 2007.

${ }^{36}$ Tajmar, M. et al.: Search for Frame Dragging in the Vicinity of Spinning Superconductors, http://arxiv.org/abs/0707.3806v5, 14 September 2007, 14 pp. Note: This paper contains a comparison with the measurements by R.D. Graham et al. and also with the Stanford-NASA Gravity Probe B experiment.

${ }^{37}$ Graham, R. D. et al.: Experiment to Detect Frame Dragging in a Lead Superconductor, www2.phys.canterbury.ac.nz/ physrin/papers/SuperFrameDragging2007.pdf), 6 July 2007, 11 pp.

${ }^{38}$ Tajmar, M. et al.: Anomalous Fiber Optic Gyroscope Signals Observed Above Spinning Rings at Low Temperature, http://arxiv.org/abs/gr-qc/0603033, June 2008.

${ }^{39}$ Tajmar, M. : Homopolar artificial gravity generator based on frame dragging, Acta Astonautica (2009) doi: 10.1016/j.actaastro.2009.10.22, 5 pp.

${ }^{40}$ Tajmar, M., F. Plecescu: Fiber Optic Gyroscope Measurements Close to Rotating Liquid Helium, to be published by American Institute of Physics, 2010, 6 pp.

${ }^{41}$ Kiefer, C.: Quantum Gravity, Oxford University Press 2007.

${ }^{42}$ Ciufolini, I. et al.: Determination of frame-dragging using earth gravity models from CHAMP and GRACE, New Astronomy 11 (2006) 527-550.

${ }^{43}$ Veltmann, C.: Facts and Mysteries in Elementary Particle Physics, World Scientific 2003.

${ }^{44}$ Zwiebach, R.: Introduction to String Theory, Cambridge Univ. Press 2009, 2nd ed.

${ }^{45}$ Dröscher,W., J. Hauser: Guidelines For a Space Propulsion Device Based on Heim's Quantum Theory, AIAA 2004-3700, 40th AIAA/ASME/SAE/ASE, Joint Propulsion Conference \& Exhibit, Fort Lauderdale, FL, 11-14 July 2004, 31 pp.

${ }^{46}$ Dröscher,W., J. Hauser: Heim Quantum Theory for Space Propulsion Physics, AIP, STAIF, 2005, 10pp.

${ }^{47}$ Dröscher,W., J. Hauser: Magnet Experiment to Measuring Space Propulsion Heim-Lorentz Force, AIAA 2005-4321, 41st AIAA/ASME/SAE/ASE, Joint Propulsion Conference \& Exhibit, Tuscon, Arizona, 10-13 July 2005, 10 pp.

${ }^{48}$ Dröscher,W., J. Hauser: Gravitational Field Propulsion, AIAA 2009-5068, 45th AIAA/ASME/SAE/ASE, Joint Propulsion Conference \& Exhibit, Denver, CO, 2-5 August 2009. 